\title{
Analytical solution for describing the thermo-mechanical behavior of plane energy geostructures
}

\author{
Alessandro F. Rotta Loria ${ }^{1, *}$, Jacopo Zannin ${ }^{2}$, Qazim Llabjani ${ }^{2}$ and Lyesse Laloui ${ }^{2}$ \\ ${ }^{1}$ Northwestern University, Department of Civil and Environmental Engineering, Mechanics and Energy Laboratory, 2145 Sheridan Road, \\ Evanston, Illinois 60208, USA \\ ${ }^{2}$ Swiss Federal Institute of Technology in Lausanne, Soil Mechanics Laboratory, Station 18, GC Building, 1015 Lausanne, CH
}

\begin{abstract}
Over the past twenty years, a substantial amount of research has been performed to expand the modeling capabilities of energy geostructures: innovative earth-contact structures that provide combined structural support and renewable energy supply. Several analytical and semi-analytical solutions have been made available for describing the thermo-mechanical behavior of cylindrical energy geostructures such as energy piles. However, no analytical solutions for capturing the thermo-mechanical behavior of plane energy geostructures such as energy walls and slabs have been developed until recently. This paper summarizes the essential features of the first analytical solution available for describing the thermo-mechanical behavior of plane energy geostructures. This analytical solution extends Winkler's model for a beam resting on an elastic soil mass to non-isothermal conditions, allowing to quantify the effects of temperature variations, axial loads, transversal loads and bending moments applied to plane energy geostructures. Such an analytical solution can be used in the analysis of an elementary unit represented by a single beam as well as complex plane energy geostructures using the superposition principle. Based on the preceding capabilities, the proposed analytical solution may effectively serve the analysis or design of plane energy geostructures under the influence of mechanical and/or thermal loads.
\end{abstract}

\section{Introduction}

Over the past twenty years, a substantial amount of research has been performed to expand the modeling capabilities of energy geostructures: innovative earthcontact structures that integrate the role of the structural support with that of the geothermal heat exchanger. The reason for this is that mechanical and thermal loads are applied to such geostructures and include unprecedented scientific and engineering challenges from geotechnical, structural and energy perspectives [1].

Numerical models of varying complexities have been historically used to address the thermo-mechanical behavior of energy geostructures subjected to mechanical and thermal loads. Computational capabilities are rising globally and make numerical modeling accessible and relatively handy in many scientific circumstances. Nevertheless, these capabilities are often unavailable in common engineering circumstances. This fact, coupled with the significant time and expertise that are required to address through computer simulations the multiphysical phenomena involved with energy geostructures, often makes the numerical modeling of such problems daunting for the engineering practice. In this context, paramount tools for the analysis and design of energy geostructures are analytical solutions.

Analytical and semi-analytical solutions have been increasingly developed in recent years for effectively describing the thermo-mechanical behavior of energy geostructures. The developed analytical models have primarily addressed the modeling of cylindrical energy geostructures such as single energy piles [2-7] and groups energy piles [8-12], leaving uncovered the modeling capabilities of other plane energy geostructures such as energy walls and slabs. Only recently an analytical solution for capturing the thermo-mechanical behavior of plane energy geostructures has been proposed [13].

This paper expands on the first analytical solution available for capturing the thermo-mechanical behavior of plane energy geostructures, describing some of its features succinctly. This analytical solution extends to non-isothermal conditions Winkler's model for a beam resting on an elastic soil mass [14], allowing to quantify the effects of temperature variations, axial loads, transversal loads and bending moments applied to plane energy geostructures. Based on this capability, the proposed model can serve as an effective alternative to numerical approaches to model the thermo-mechanical behavior of energy geostructures.

In the following, a framework about the effects of thermal and mechanical loads on the thermo-mechanical behavior of plane energy geostructures is proposed. Next, the essentials of the proposed analytical solution are reported. Then, a discussion of the capabilities of such an analytical solution and one application example is presented. Finally, closure is reported.

\footnotetext{
* Corresponding author: af-rottaloria@northwestern.edu
} 


\section{Theoretical framework}

Thermal and mechanical loads applied to energy geostructures result in a variety of effects for the structure and the ground. These effects deserve consideration in the geotechnical and structural analysis and design of such geostructures [15].

Thermal loads are associated with temperature variations applied within and around energy geostructures [13]. These variations are usually non-uniform and can be idealized as composed of two contributions: a constant distribution of temperature variation, $\Delta T_{a}$, inducing an axial effect, and a linear distribution of temperature variation, $\Delta T_{c}$, inducing a bending effect. Constant distributions of temperature variations are typically considered alone when addressing the analytical modeling of cylindrical energy geostructures. Constant and linear variations of temperature variations are typically considered together when addressing the analytical modeling of plane energy geostructures. The axial and bending effects resulting from these distributions can be associated with a thermally induced axial strain and curvature, respectively. Prevention of these effects results in axial loads and bending moments, respectively.

Mechanical loads are associated with forces and displacements applied to energy geostructures [13]. The effects of these forces and displacements are axial and transversal displacements, neutral axis rotations, shear forces and bending moments. The concept of degree of freedom is powerful to address the effects caused by temperature variations applied to energy geostructures: it expresses the development of a relevant physical quantity to its value under free thermal deformation conditions. In the following, this concept is used to address axial and flexural effects caused by thermal loads applied to energy geostructures. A constant distribution of temperature variation, $\Delta T_{a}$, applied along an energy geostructure free to move at its ends causes a free thermally induced axial strain, $\varepsilon_{f}^{\text {th }}$, as

$$
\varepsilon_{f}^{t h}=-\alpha \Delta T_{a}
$$

where $\alpha$ is the linear thermal expansion coefficient of the material. A linear distribution of temperature variation applied along an energy geostructure free to move at its ends causes the development of a free thermally induced curvature, $\chi_{f}^{\text {th }}$, as

$$
\chi_{f}^{t h}=-\frac{\alpha}{I} \int_{A} y_{h} \frac{2 \Delta T_{c}}{h} d A=-\frac{2 \alpha \Delta T_{c}}{h}
$$

where $I$ is the moment of inertia of the cross-section $A$, and $y_{h}$ is the coordinate along the structure height $h$. A partial restraint applied to an energy geostructure by any given boundary condition yields to the development of an observed axial deformation and curvature that are a fraction of the ones under free thermal deformation conditions. This fact involves the development of only a portion of axial deformation and curvature with respect to free deformation conditions, while a portion of axial deformation and curvature is blocked.
The preceding considerations yield to the definition of a degree of freedom associated with axial effects

$$
D O F_{a}=\varepsilon_{o}^{t h} / \varepsilon_{f}^{t h} \quad 0 \leq D O F_{a} \leq 1
$$

and a degree of freedom associated with flexural effects

$$
D O F_{c}=\chi_{o}^{t h} / \chi_{f}^{t h} \quad 0 \leq D O F_{c} \leq 1
$$

where $\varepsilon_{o}^{\text {th }}$ and $\chi_{o}^{\text {th }}$ are the observed thermally induced axial strain and curvature, respectively. Internal actions develop as a consequence of the blocked portion of deformations. The restraint of a constant distribution of temperature variation causes a thermally induced axial force. The restraint of linear distribution of temperature variation causes a thermally induced bending moment. The observed thermally induced axial force, $N_{o}^{t h}$, and bending moment, $M_{o}^{t h}$, caused by such temperature variations can be quantified as

$$
\begin{aligned}
& N_{o}^{t h}=N^{t h}=\mathrm{A} E \alpha \Delta T_{a}\left(1-D O F_{a}\right) \leq N_{b}^{t h} \\
& M_{\mathrm{o}}^{t h}=M^{t h}=E I \frac{2 \alpha \Delta T_{c}}{h}\left(1-D O F_{c}\right) \leq M_{b}^{t h}
\end{aligned}
$$

where $N_{b}^{t h}$ and $M_{b}^{t h}$ are the axial force and bending moment under completely blocked deformation conditions, respectively. Any distribution of axial force and bending moment in the orthogonal direction considered thus far could be addressed at any time by applying the same mathematical along such direction.

\section{The analytical solution}

The analytical solution expanded hereafter has been developed for addressing the thermo-mechanical behavior of plane energy geostructures by Zannin et al. [13]. This solution resorts to the so-called subgrade reaction method initially developed by Winkler [14] to model plane geostructures (e.g., beams) resting on an elastic soil mass under isothermal conditions. A schematic of this approach is reported in Figure 1. Under non-isothermal conditions, the subgrade reaction method can address the axial and flexural effects of thermal and mechanical loads on the response of energy walls, slabs and cut-and-cover tunnels.

From Euler-Bernoulli theory, a relationship between the deflection and the loads characterizing any plane geostructure (e.g., beam) can be written for obtaining the fourth-order differential equation that governs the problem. In small deformations, Euler-Bernoulli theory involves that straight lines or planes normal to the neutral axis of the beam remain straight and normal to the considered axis after deformation. This feature allows expressing the bending moment proportionally to the second derivative of the deflection.

Based on the preceding premises, Winker's solution resorts to the three following hypotheses. (i) The subgrade reaction modulus, $k_{s}$, is independent of the pressure and involves the same response for both loading and unloading. (ii) The value of $k_{s}$ does not vary in space (for non-uniform soil deposits, $k_{s}$ should be averaged 
depending on the thickness and the mechanical characteristics of the layers). (iii) The springs work unidirectionally and independently of each other. The previous hypotheses involve the following practical considerations. (i) The soil follows a linear elastic behavior, making the solution representative and suitable for the analysis of limited deformation levels (so-called serviceability conditions). (ii) The soil reaction is uniformly distributed among the springs, making the solution unsuitable to describe rigid beams but particularly appropriate to model flexible beams. (iii) Any influence caused by loads in the soil outside the beam length cannot be captured, making the solution suitable to provide accurate estimates of action effects along the beam only.

(a) - Hinged

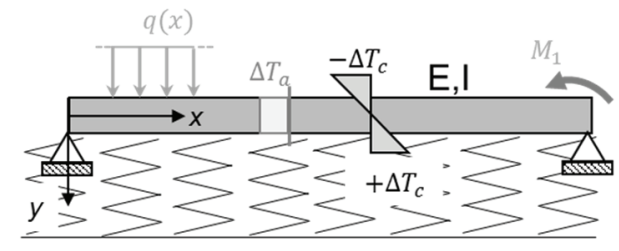

(b) - Fixed

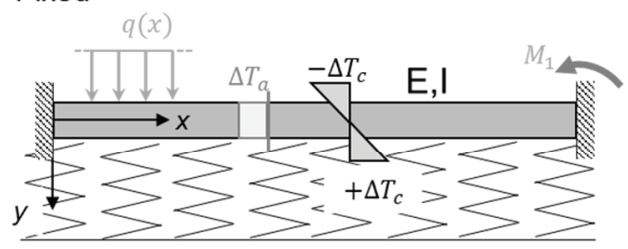

(c) - Partly restrained

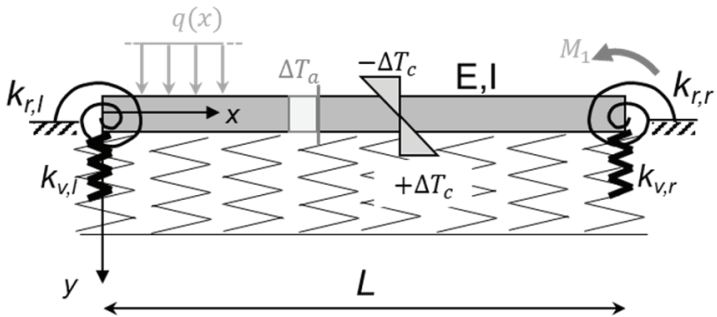

Fig. 1. Theoretical principle characterizing the subgrade reaction method for different boundary conditions: (a) hinged, (b) fixed and (c) partly restrained (by transversal and rotational springs of stiffness $\left(k_{v, l}, k_{v, r}\right)$ and $\left(k_{r, l}, k_{r, r}\right)$, respectively).

According to the previous considerations, the rotation, $\theta(x)$, the bending moment, $M(x)$ and the shear force, $V(x)$, characterizing any infinitely small element of a beam as a consequence of the application of thermal and mechanical loads inducing flexural effects (e.g., nonuniform distribution of temperature variations or distributed loads perpendicular to the beam axis) respectively read

$$
\begin{gathered}
\theta(x) \approx \tan \theta(x)=\frac{d y(x)}{d x} \\
M(x)=-E I \frac{d^{2} y(x)}{d x^{2}}-E I \chi_{f}^{t h}
\end{gathered}
$$

$$
V(x)=\frac{d M(x)}{d x}
$$

where $x$ is the coordinate axis along the beam axis, $\theta$ is the angle between the axis of the beam in its deformed configuration and the horizontal line, $y$ is the deflection of the beam and $E$ is the Young's modulus of the beam. In addition to the previous actions, axial forces, $N$, can characterize any element of a beam due to the application of thermal and mechanical loads (e.g., uniform distributions of temperature variations or mechanical forces applied normal to the beam transversal crosssection). Consideration of one-dimensional conditions involves neglecting any extension or contraction of the beam within its cross-section due to the considered actions. The present model can be formulated by analyzing the equilibrium of a beam element resting on an elastic soil of length $d x$ in its deformed configuration (Figure 2(a)).

(a)
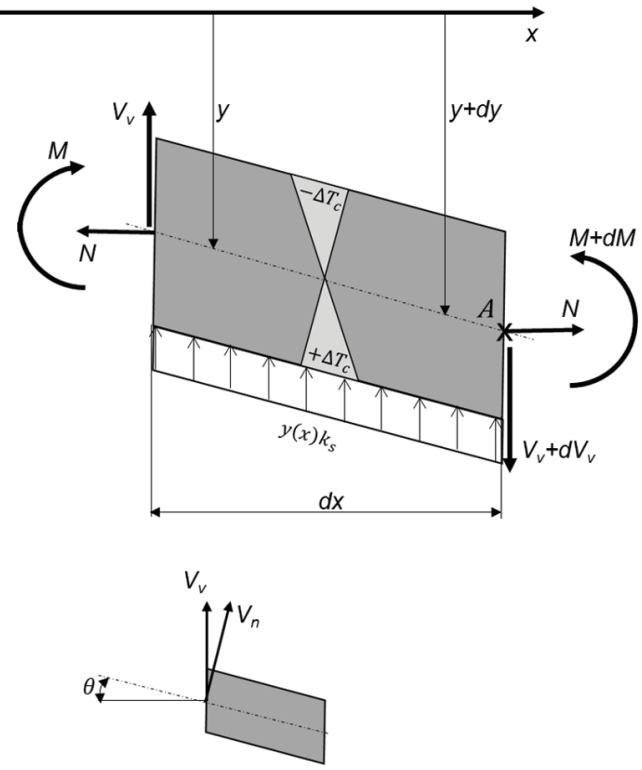

Fig. 2. Schematic of (a) the equilibrium of a beam element and (b) the geometric decomposition of shear actions.

Vertical equilibrium gives

$$
\frac{d V_{v}}{d x}=y(x) k_{s}
$$

where $V_{v}$ is the vertical shear force, which can be correlated to the normal shear force, $V_{n}$ (Figure 2(b)):

$$
V_{n}=V_{v} \cos \theta-N \sin \theta \approx V_{v}-N \frac{d y}{d x}
$$

The generalized shear action is evaluated by modifying equation (9) to account for the two components of the shear force. The following expression is obtained 


$$
\begin{array}{r}
V(x)=-E I \frac{d^{3} y(x)}{d x^{3}}-E I \frac{d \chi^{t h}}{d x} \\
+N \frac{d y(x)}{d x}
\end{array}
$$

The moment equilibrium around point $\mathrm{A}$, divided by $d x$, can be written as follows (neglecting second-order terms):

$$
\frac{d M}{d x}=V_{v}-N \frac{d y}{d x}
$$

Substituting equation (8) into (13), differentiating with respect to $x$ and considering equation (10), the differential equation of the elastic line is obtained:

$$
\begin{gathered}
E I\left(\frac{d^{4} y}{d x^{4}}+\frac{d \chi^{t h^{2}}(x)}{d x^{2}}\right)-N \frac{d^{2} y(x)}{d x^{2}} \\
+k_{s} y(x)=0
\end{gathered}
$$

Equation (14) has a solution of the type:

$$
\begin{aligned}
y(x)=\left(C_{1} e^{\alpha^{*} x}\right. & \left.+C_{2} e^{-\alpha^{*} x}\right) \cos \beta^{*} x \\
& +\left(C_{3} e^{\alpha^{*} x}\right. \\
& \left.+C_{4} e^{-\alpha^{*} x}\right) \sin \beta^{*} x
\end{aligned}
$$

where $C_{1}, C_{2}, C_{3}$ and $C_{4}$ are integration constants, and

$$
\begin{aligned}
& \alpha^{*}=\sqrt{\lambda^{2}+\frac{N}{4 E I}} \\
& \beta^{*}=\sqrt{\lambda^{2}-\frac{N}{4 E I}}
\end{aligned}
$$

where $\lambda$ is called the characteristic of the system. This parameter can be determined as

$$
\lambda=\sqrt[4]{\frac{k_{S}}{4 E I}}
$$

The integration constants $C_{1}, C_{2}, C_{3}$ and $C_{4}$ can be defined by imposing the boundary conditions and solving the system of equations (7), (8) and (15). The resolution of this system with appropriate boundary conditions allows extending the classical Winkler's solution to nonisothermal conditions [13].

\section{Capabilities}

The obtained analytical solution can effectively model the effects caused by arbitrary thermal and mechanical loads applied to simple plane geometries: geostructures (e.g., beams) of finite dimensions resting on a Winkler-type soil mass that are arbitrarily restrained by boundary conditions and subjected to loading. This capability resorts to the superposition principle - an essential constituent of the theory employed herein. Based on this principle, the effects caused by arbitrary combinations of loads can be considered as the sum of the effects caused by single loads solved separately. Accordingly, the deflection, rotation, bending moment, shear force and normal force can be considered as made of two contributions: one component caused by any thermal load and another component caused by any mechanical load.

The obtained analytical model can also effectively address the effects of arbitrary combinations of thermal and mechanical loads on complex plane geometries: geostructures composed by multiple elements mutually connected one another (e.g., cut-and-cover structures, structures involving wall-slab and wall-anchors intersections, mat foundations and multi-floored structures) that are partly or entirely surrounded by a Winkler-type soil mass and subjected to loading. In the present context, this capability is again related to the superposition principle. Relevant simple plane geometries can be considered as an elementary unit for more complex plane geometries. The effects of thermal and mechanical loads applied to these geometries can be addressed straightforwardly. Once such effects are obtained, they can be considered as boundary loads for the other unit(s) composing the complex plane geometry. From the foregoing, general combinations of thermal and mechanical loads can be solved for all the elementary units constituting any complex geometry, thus yielding a complete procedure for addressing the related response (Fig. 3).

\section{Application example}

In the following, an application of the developed analytical solution to a single plane geometry constituted by a beam with hinged and partly restrained boundary conditions is proposed. Hinged conditions aim at representing the behavior of beams characterized by connections with an infinite transversal stiffness and zero rotational stiffness at their ends. Such conditions do not usually characterize plane energy geostructures in practice. Partly restrained conditions aim at representing the behavior of beams connected with other structural elements that are common in practice (e.g., wall-slab connections, such as for cut-and-cover structures).

In the following, a beam characterized by a length $L=10 \mathrm{~m}$, a height $h=0.5 \mathrm{~m}$, and a breadth $B=1 \mathrm{~m}$ is considered to be subjected to a uniform distribution of temperature variation of $\Delta T_{c}=1.0^{\circ} \mathrm{C}$. The rationale for considering this temperature distribution is that it provides a unitary response to the modeled problem. In particular, as long as the hypothesis of a reversible response of the soil (and structure) holds, temperature variations of $\Delta T_{c}=10.0{ }^{\circ} \mathrm{C}$ and $\Delta T_{c}=-1.0{ }^{\circ} \mathrm{C}$, for example, will yield to results that are ten times higher and opposite compared to those discussed here, respectively. 

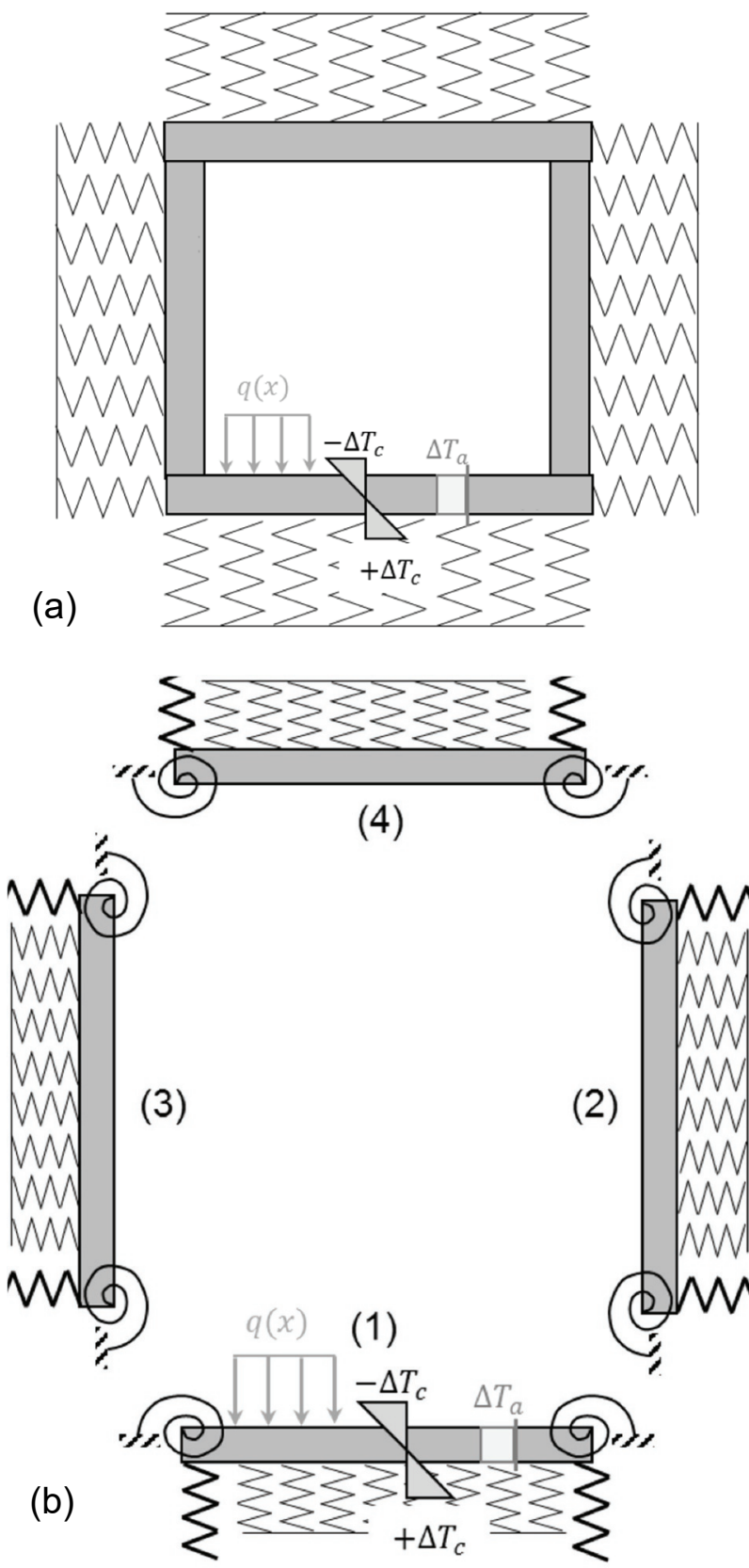

Fig. 3. Theoretical principle characterizing the subgrade reaction method: (a) the problem and (b) solution approach based on the elastic superposition principle (consisting of the successive and interconnected resolution of the equilibrium of the structural elements (1)-(4)).

When considering partly restrained conditions, symmetric boundary conditions at the ends of the beam are considered: $k_{v, l}=k_{v, r}=k_{v}=1.0 \times 10^{8}$ and $k_{r, l}=$ $k_{r, r}=k_{r}=1.0 \times 10^{9}$. For both of the considered boundary conditions, the subgrade reaction modulus reads $k_{s}=10^{6} \mathrm{~N} / \mathrm{m}^{3}$, aiming at addressing soft soils. The Young's modulus of the beam is $E=25 \mathrm{GPa}$.

To validate the capabilities of the present analytical model in addressing the considered problem, reference numerical simulations have been run (the details of the numerical models are described by Zannin et al. [13]). Specifically, two problems have been numerically simulated: (i) a beam resting on a spring foundation and (ii) a beam resting on a continuum soil mass. In the first case, the analytical and numerical modeling results perfectly match (error of $0 \%$ ), as they describe the same problem. This result fully validates the correctness of the proposed extension of Winkler's solution in capturing the effects of thermal and mechanical actions applied to plane energy geostructures. In the second case, differences between the analytical and numerical modeling results are observed due to the differences between the considered modeling approaches. These differences are discussed hereafter.

The comparison between analytical and numerical modeling results is reported in Fig. 4. For both boundary conditions, the maximum deflection takes place at the midpoint of the beam. The maximum values of rotation are recorded at the beam ends. Zero rotation is evaluated at the mid-span of the beam, showing an anti-symmetric distribution. Hinged conditions allow for free rotation at the boundaries and null vertical deflection. Under these conditions, the beam displaces downwards, showing a maximum at its mid-span and zero deflection at the boundaries. Rotation is maximum (in absolute value) at the boundaries and goes to zero at the mid-span. At the considered location, the deflection presents a minimum. Partly restrained conditions affect the magnitude of the deflection and rotation. The deformed shape is the same as for hinged conditions but characterized by a lower magnitude.

The differences between the analytical and numerical modeling results are higher for the hinged case compared to the partly restrained case. If there is some rotational stiffness at the boundaries, as it can be expected for most practical applications of plane energy geostructures, the results of the analytical model approach the numerical ones. Therefore, the developed analytical solutions may be considered a valuable tool to develop simplified investigations on the response of plane energy geostructures subjected to thermal and mechanical loads.

\section{Closure}

This study aims at taking a step forward towards an assessment of the thermo-mechanical behavior of plane energy geostructures. From this perspective, the present work describes critical features of the first analytical model available for addressing the influence of thermal and mechanical loads applied to plane energy geostructures. For the first time, such a model discretizes plane energy geostructure as a beam lying on a Winklertype soil mass under non-isothermal conditions. As a result Winkler's solutions is extended to capture the influence of generally distributed thermal and mechanical loads applied to relatively simple and complex plane geostructures.

Comparisons between the results of the developed analytical solution and numerical simulations highlight relatively close and valuable predictions for investigating the behavior of plane energy geostructures under non- 
isothermal conditions, especially when considering partly restrained boundary conditions that are diffused in practice. Based on such a result, the present analytical solution could be used for developing effective yet informative schematic analyses and designs of plane energy geostructures subjected to thermal and mechanical loads, as well as for running handy parametric studies.
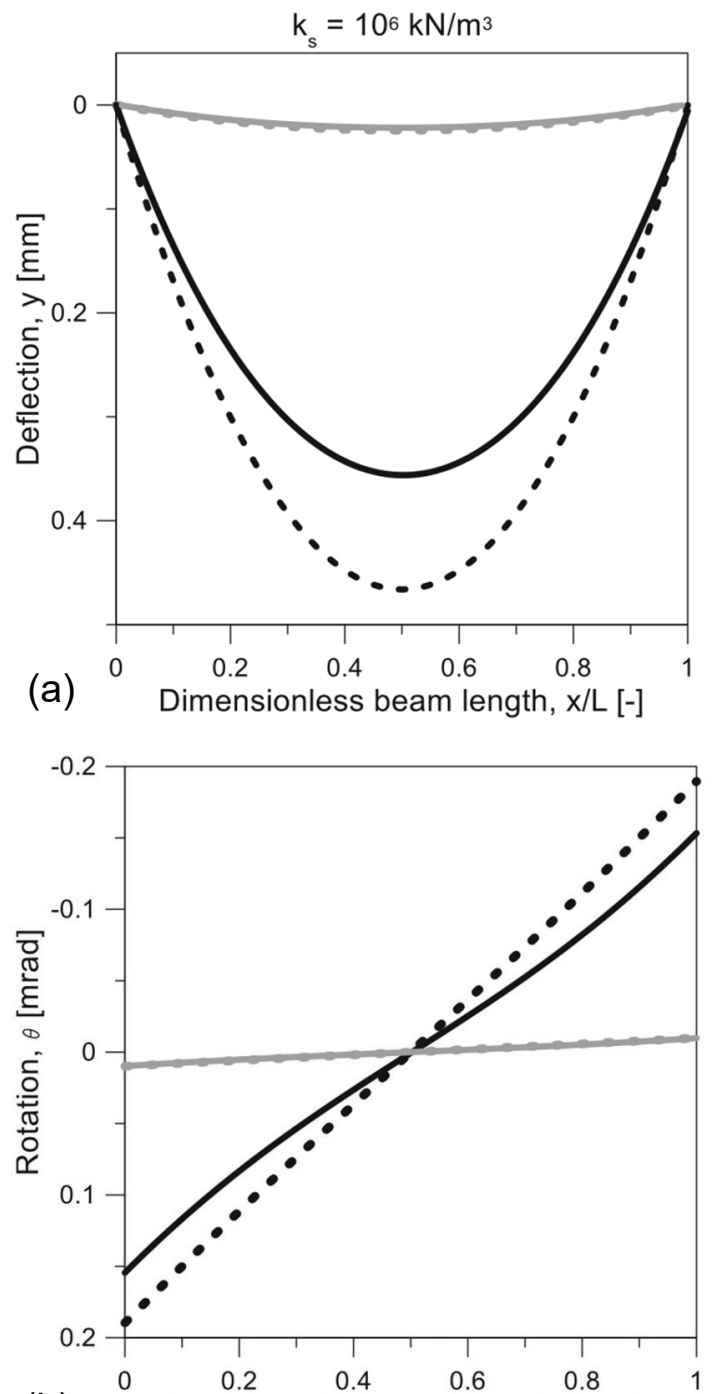

(b) Dimensionless beam length, $\mathrm{x} / \mathrm{L}[-]$

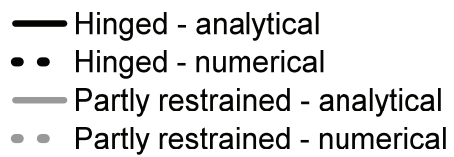

Fig. 4. Comparison between analytical and numerical predictions of the thermo-mechanical response of a plane energy geostructure subjected to a linear distribution of temperature variation: (a) deflection and (b) rotation.

\section{References}

[1] Laloui L, Rotta Loria AF. Analysis and Design of Energy Geostructures. Elsevier Academic Press; 2019.
[2] Knellwolf C, Peron H, Laloui L. Geotechnical analysis of heat exchanger piles. Journal of Geotechnical and Geoenvironmental Engineering 2011;137:890-902.

[3] Suryatriyastuti M, Mroueh H, Burlon S. A load transfer approach for studying the cyclic behavior of thermo-active piles. Computers and Geotechnics 2014;55:378-91.

[4] Pasten C, Santamarina JC. Thermally induced longterm displacement of thermoactive piles. Journal of Geotechnical and Geoenvironmental Engineering 2014;140:06014003.

[5] Chen D, McCartney JS. Parameters for Load Transfer Analysis of Energy Piles in Uniform Nonplastic Soils. International Journal of Geomechanics 2016:04016159.

[6] Sutman M, Olgun CG, Laloui L. Cyclic load-transfer approach for the analysis of energy piles. Journal of Geotechnical and Geoenvironmental Engineering 2019;145:04018101.

[7] Iodice C, Di Laora R, Mandolini A. Analytical Solutions for Ultimate Limit State Design of Thermal Piles. Journal of Geotechnical and Geoenvironmental Engineering 2020;146:04020016.

[8] Rotta Loria AF, Laloui L. The equivalent pier method for energy pile groups. Géotechnique 2017;67:691702.

[9] Rotta Loria AF, Vadrot A, Laloui L. Analysis of the vertical displacement of energy pile groups. Geomechanics for Energy and the Environment 2018;16:1-14.

[10] Rotta Loria AF, Vadrot A, Laloui L. Effect of nonlinear soil deformation on the interaction among energy piles. Computers and Geotechnics 2017;86:920.

[11] Ravera E, Sutman M, Laloui L. Analysis of the interaction factor method for energy pile groups with slab. Computers and Geotechnics 2019:103294. https://doi.org/10.1016/j.compgeo.2019.103294.

[12] Ravera E, Sutman M, Laloui L. Load Transfer Method for Energy Piles in a Group with Pile-SoilSlab-Pile Interaction. Journal of Geotechnical and Geoenvironmental Engineering 2020;146:04020042.

[13]Zannin J, Rotta Loria AF, Llabjani Q, Laloui L. Extension of Winkler's solution to non-isothermal conditions for capturing the behaviour of plane geostructures subjected to thermal and mechanical actions. Computers and Geotechnics 2020:In print.

[14] Winkler E. Die Lehre von der Elasticitaet und Festigkeit: mit besonderer Rücksicht auf ihre Anwendung in der Technik für polytechnische Schulen, Bauakademien, Ingenieue, Maschinenbauer, Architecten, etc. Dominicus; 1867.

[15] Rotta Loria AF, Bocco M, Garbellini C, Muttoni A, Laloui L. The role of thermal loads in the performance-based design of energy piles. Geomechanics for Energy and the Environment 2020;21:100153. 